\title{
Superhydrophobic polymer with hierarchical porous structure as novel QCM sensing material for toluene/water selectively detection
}

\author{
Luyu Wang, Jiaqiang $X u^{*}$ \\ NEST Lab, Department of Physics, Department of Chemistry, College of Science, Shanghai University, \\ Shanghai, 200444, P. R. China \\ Corresponding author's e-mail address: xujiaqiang@shu.edu.cn
}

\begin{abstract}
:
In this work, a superhydrophobic polymer with hierarchical porous structure is firstly used as novel Quartz crystal microbalance (QCM) sensing material for toluene detection. Superhydrophobic property, porous structure, and rich aromatic ring composition of the polymer make its stable toluene/water selectively detection performance. The sensor exhibits good sensitivity for toluene detection. The response remain stable at different ambient humidity. The detection limit reaches down to $1 \mathrm{ppm}$, and the selectivity is satisfactory to twelve kinds of common interfering gases. This superhydrophobic porous polymer functionalized QCM sensor may be a potential candidate for the future application of toluene detection.
\end{abstract}

Key words: Quartz crystal microbalance, Toluene, Gas sensor, Porous polymer, Superhydrophobicity

\section{Results and discussion of characterization}

The polymer is made up of PDVB and $\mathrm{SiO}_{2}$. The textural property of the polymer was first characterized by nitrogen adsorption. The polymer exhibits high BET surface area (818.9 $\left.\mathrm{m}^{2} \mathrm{~g}^{-1}\right)$ and pore volume $\left(1.57 \mathrm{~cm}^{3} \mathrm{~g}^{-1}\right)$. As shown in Fig. 1, the nitrogen adsorptiondesorption isotherms of polymer behave as a typical $\mathrm{H} 4$ hysteresis loop, indicating its nanoporous structures. A slow increase in the adsorption amount occurs in the relative pressure range of $0.4-0.8$, indicating the presence of mesopores [1]. As shown in the insret of Fig. 1, the pore size distribution of the polymer reveals the hierarchical porosity mainly ranges from 10-100 $\mathrm{nm}$. TEM image of the polymer (Fig. 2) indicates the presence of imesoporous, which is consistent with nitrogen adsorption techniques. The contact angle test proves its superhydrophobicity.

\section{Results and discussion of toluene sensing properties}

In this section, toluene sensing properties of polymer functionalized QCM sensor are discussed. According to Fig. 3, quick response and high sensitivity of the polymer functionalized sensor are verified by successive recording of five response cycles toward higher concentration of toluene from $5 \mathrm{ppm}$ to 200 ppm, and the whole live detecting is carried out within $1000 \mathrm{~s}$. As the concentration of toluene is increasing in the range from $5 \mathrm{ppm}$ to $200 \mathrm{ppm}$, the QCM frequency-response value is also increased. The inset of Fig. 3 shows the response curve towards $1 \mathrm{ppm}$ toluene, we find that the response can achieve $8 \mathrm{~Hz}$, indicating that the limit of detection of the polymer functionalized sensor is lower than $1 \mathrm{ppm}$.

As shown in Fig. 4, the response towards 5 ppm toluene under different $\mathrm{RH}$ of $35 \%, 45 \%$, $55 \%, 65 \%, 75 \%, 85 \%$, and $90 \%$ are almost unchanged. This experimental result indicates the superior toluene/water selective detecting performance of the polymer functionalized sensor.

As shown in Fig. 5, the contrastive responses of polymer functionalized QCM sensor are listed upon exposure to toluene vapor (25 ppm) and other twelve kinds of vapours (25 ppm). When the sensor is exposed to these vapours, it shows the highest frequency change of 171 $\mathrm{Hz}$ towards toluene. It is obvious that polymer functionalized sensor possesses a desirable detection capacity towards toluene because of the reversible " $\pi-\pi$ conjugated interation" between the polymer and BTEX, such as 
toluene. This affinity extremely enhances the selectivity of the QCM sensor. All of the above tests demonstrate that the sensing performance of this sensor is desirable.

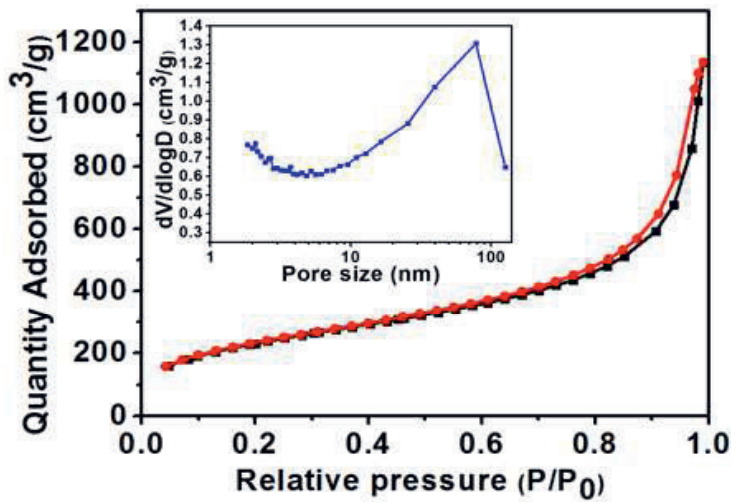

Fig. 1. $N_{2}$ isotherms and (b) pore size distribution of the polymer.

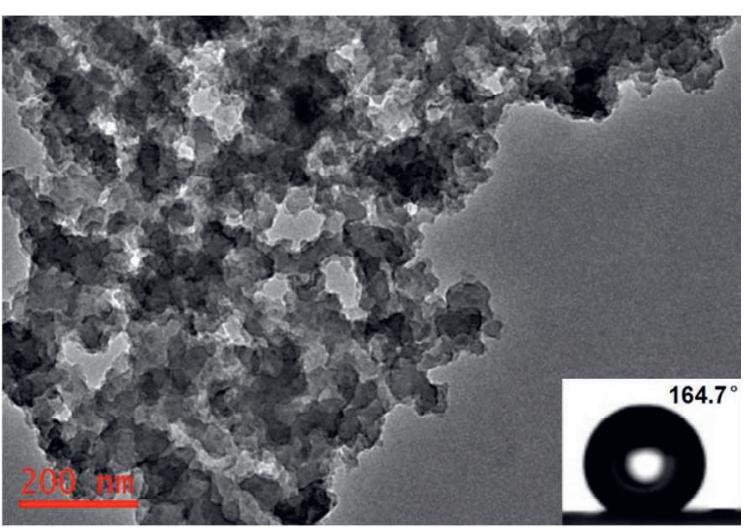

Fig. 2. The TEM image of the superhydrophobic polymer.

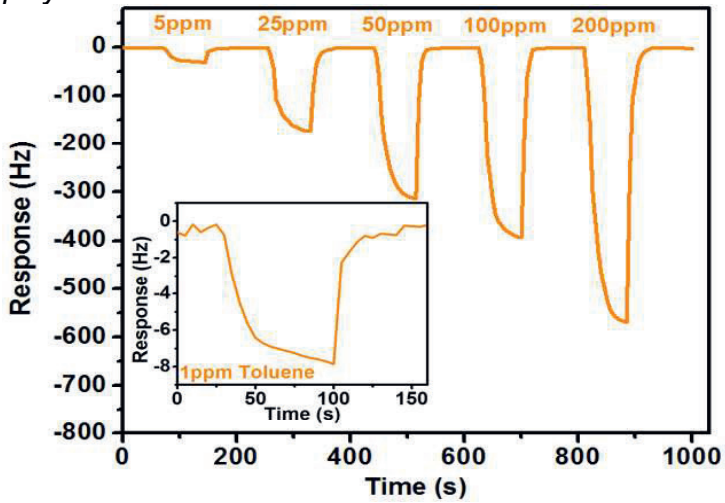

Fig. 3. Typical sensor responses versus toluene of various concentrations of $5,25,50,100,200 \mathrm{ppm}$, as well as $1 \mathrm{ppm}$.

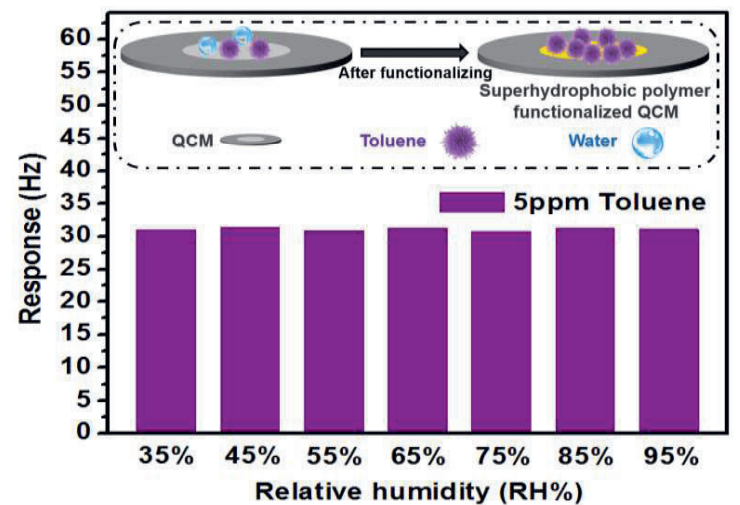

Fig. 4. The response of the polymer towards $5 \mathrm{ppm}$ toluene under defferent relative humidity.

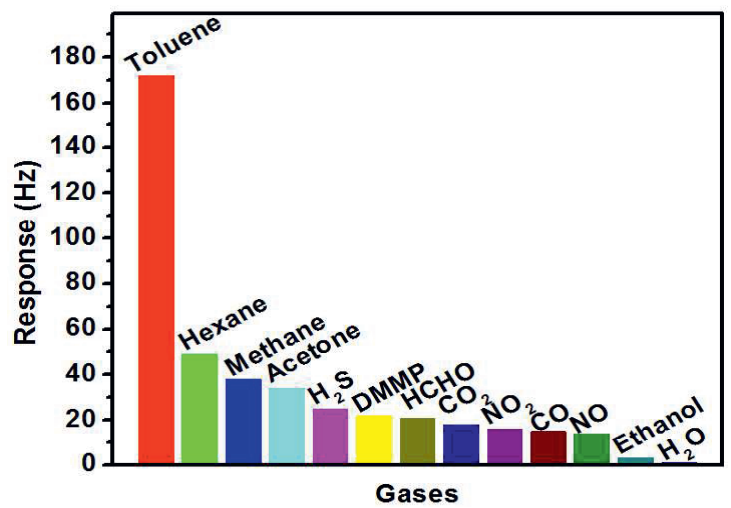

Fig. 5. Responses of the sensor to various kinds of interfering gases compared with that of 20 ppm toluene.

\section{References}

[1] Y. L. Zhang, J. N. Wang, Y. He, Y. Y. He, B. B. Xu, S. Wei, F. S. Xiao, Solvothermal Synthesis of Nanoporous Polymer Chalk for Paintis, Langmuir 27, 12585-12590 (2011); doi: 10.1021/la2018264 